\title{
Imaging modalities in pregnant cancer patients
}

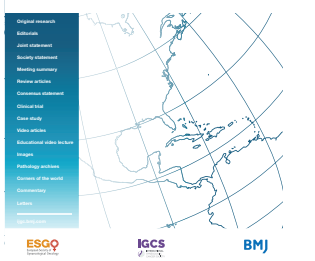

(2)

\section{OPEN ACCESS}

For numbered affiliations see end of article.

Correspondence to

Professor Vincent Vandecaveye, Leuven, Belgium; vincent. vandecaveye@uzleuven.be

Received 18 September 2020 Revised 19 0ctober 2020 Accepted 21 0ctober 2020

\author{
Vincent Vandecaveye (ID , , ${ }^{1,2}$ Frédéric Amant, ${ }^{3,4,5}$ Frédéric Lecouvet, ${ }^{6}$ Kristel Van Calsteren, ${ }^{3,7}$ \\ Raphaëla Carmen Dresen ${ }^{1,2}$
}

\section{ABSTRACT}

Cancer during pregnancy is increasingly diagnosed due to the trend of delaying pregnancy to a later age and probably also because of increased use of non-invasive prenatal testing for fetal aneuploidy screening with incidental finding of maternal cancer. Pregnant women pose higher challenges in imaging, diagnosis, and staging of cancer. Physiological tissue changes related to pregnancy makes image interpretation more difficult. Moreover, uncertainty about the safety of imaging modalities, fear of (unnecessary) fetal radiation, and lack of standardized imaging protocols may result in underutilization of the necessary imaging tests resulting in suboptimal staging. Due to the absence of radiation exposure, ultrasound and MRI are obvious first-line imaging modalities for detailed locoregional disease assessment. MRI has the added advantage of a more reproducible comprehensive organ or body region assessment, the ability of distant staging through whole-body evaluation, and the combination of anatomical and functional information by diffusion-weighted imaging which obviates the need for a gadolinium-based contrast-agent. Imaging modalities with inherent radiation exposure such as CT and nuclear imaging should only be performed when the maternal benefit outweighs fetal risk. The cumulative radiation exposure should not exceed the fetal radiation threshold of 100 mGy. Imaging should only be performed when necessary for diagnosis and likely to guide or change management. Radiologists play an important role in the multidisciplinary team in order to select the most optimal imaging strategies that balance maternal benefit with fetal risk and that are most likely to guide treatment decisions. Our aim is to provide an overview of possibilities and concerns in current clinical applications and developments in the imaging of patients with cancer during pregnancy.

\section{INTRODUCTION}

Cancer during pregnancy is relatively rare and is diagnosed in approximately 1:1000-2000 pregnancies. ${ }^{1}$ Its incidence is likely to rise by the trends of delaying pregnancy to more advanced maternal age. Additionally, the implementation of genome-wide non-invasive prenatal testing for fetal aneuploidy screening will likely increase the number of incidentally found maternal malignancies. ${ }^{2}$ The most common cancer types diagnosed during pregnancy are breast cancer, hematological malignancies, gynecological malignancies, gastrointestinal cancers, and thyroid cancer and melanoma ${ }^{13}$ (Table 1). The clinical assessment of pregnant patients with cancer is difficult. The overlap of cancer-related and physiological gestational symptoms, such as fatigue, breast changes, or constipation, may delay diagnosis with disease detected in more advanced stages. ${ }^{14}$ Non-invasive prenatal testing may enable earlier pre-symptomatic detection of maternal tumors and warrants diagnostic imaging investigation to assess the possible presence of tumour. ${ }^{5}$

Aiming for the best possible oncological outcome for the mother without interfering with the development of the child, physicians adhere to treatment schedules for non-pregnant patients. In this setting oncologic treatment during pregnancy is favored over termination of pregnancy and over elective preterm delivery. ${ }^{1}$ The long term outcomes of children exposed to chemotherapy in the second or third trimester of pregnancy, seem reassuring when evaluated at the ages of 3 and 6 years. ${ }^{6}$ The ability to deliver complex but effective treatment to pregnant cancer patients requires accurate assessment of the extent of disease and treatment response, demanding meticulous diagnostic imaging. An important hurdle in imaging the pregnant cancer patient is the need to balance maternal benefit and fetal risk. This makes it difficult to standardize the diagnostic approach and harbors the risk to underutilize diagnostic imaging, thus resulting in a suboptimal diagnostic (and consequently therapeutic) management as compared with non-pregnant counterparts. Therefore, decisions on optimized diagnostic strategies should be made within the multidisciplinary team involving radiologists and nuclear imaging physicians.

Ultrasound and MRI are preferred as primary imaging modalities due to the lack of ionizing radiation and highly accurate clinical performance. CT, positron emission tomography (PET)/CT, and bone scintigraphy may be selectively performed in unresolved imaging or diagnostic dilemmas when the maternal benefit outweighs risk to the fetus. ${ }^{7}$ Our aim is to provide an overview of options in current clinical applications and developments in imaging of cancer during pregnancy.

\section{SAFETY CONSIDERATIONS}

When applied correctly, ultrasound is considered to pose no risk to the fetus. However, there 
Table 1 Distribution of cancers during pregnancy (modified after $^{1}$ )

\begin{tabular}{lcc}
\hline Tumor type & Total number & Incidence \\
\hline Breast cancer & 462 & $39 \%$ \\
Cervical cancer & 147 & $13 \%$ \\
Lymphoma & 113 & $10 \%$ \\
Ovarian cancer & 88 & $7 \%$ \\
Leukemia & 68 & $6 \%$ \\
Gastro-intestinal cancer & 49 & $4 \%$ \\
Melanoma & 46 & $4 \%$ \\
Thyroid cancer & 37 & $3 \%$ \\
Brain cancer & 21 & $2 \%$ \\
Other & 139 & $21 \%$ \\
\hline
\end{tabular}

is a theoretical risk of teratogenic effect secondary to sustained temperature elevation from prolonged ultrasound exposure, and as a result the US Food and Drug Administration (FDA) has limited the spatial-peak temporal average intensity to $720 \mathrm{~mW} / \mathrm{cm}^{2} .89$ In this context, it is important to note that ultrasound systems using nonobstetric transducers and settings - as generally used for cancer diagnosis - produce higher temperatures than systems configured for obstetric use. Color doppler and spectral doppler are more prone to risk of temperature elevation than B-mode imaging. Therefore, ultrasound should be judiciously used for indications where it is most likely to provide reproducible disease assessment and clinical benefit. Examination time should not exceed $30 \mathrm{~min}$ and the use of oppler should be limited in exposure time and output level in early pregnancy. ${ }^{10}$

The use of ionizing radiation imaging in the pregnant patient should always adhere to the rule that radiation doses should be kept as low as reasonably achievable whenever indicated. ${ }^{11}$ The risks associated with fetal exposure to ionizing radiation is the main concern in pregnant patients for which fetal outcome is determined by gestational age, radiation dose, and exposure time. Cumulative fetal radiation exposure over $100 \mathrm{mGy}$ should be avoided at any cost, as exceeding this limit can result in fetal death, growth restriction, microcephaly, and intellectual disability. ${ }^{12}$ It is important to note that all currently used ionizing radiation imaging techniques have a fetal exposure lower than $50 \mathrm{mGy}$, which has not been associated with an increase in fetal anomalies, growth restriction, or pregnancy loss. ${ }^{9}$ A single examination does not pose substantial risk to the fetus (Table 2). However, if multiple imaging studies are needed, careful monitoring of cumulative fetal radiation exposure is required in order not to inadvertently exceed the threshold. ${ }^{11}$

An additional - and possibly overlooked - risk related to in-utero radiation exposure is fetal carcinogenesis and genetic defects. The occurrence of these stochastic effects show a linear relationship with radiation dose in the absence of a dose threshold, meaning they can also occur at low-dose exposure. ${ }^{9}$ The carcinogenic risk appears to be highest during the first trimester of pregnancy and a fetal exposure of 10-20 mGy may increase the risk of leukemia by a factor 1.5-2. ${ }^{9}$ However, given the very low likelihood of developing childhood cancer after a normal pregnancy, possible increase in incidence rates will still result in a very low cumulative risk
Table 2 Fetal radiation dose for the different ionizing radiation techniques (modified after $^{79}$ )

\begin{tabular}{ll}
\hline Imaging technique & $\begin{array}{l}\text { Fetal radiation } \\
\text { dose (mGy) }\end{array}$ \\
\hline Chest X-ray & $<0.01$ \\
Mammography (two planes, bilateral) & $<0.01$ \\
CT of the head & $<0.005-0.5$ \\
CT of the chest & $0.001-0.66$ \\
CT of the abdomen/pelvis & $8-25$ \\
${ }^{99}$ Tc bone scintigraphy & 3.3 \\
${ }^{18}$ F-FDG PET/CT & $10-50$ \\
\hline
\end{tabular}

for childhood cancer and should not withhold the use of ionizing radiation imaging techniques when clinically indicated. ${ }^{13}$ Maximal reduction or avoidance of radiation exposure to the fetus is key. This can be most easily achieved by implementing imaging techniques that do not use ionizing radiation with at least equal diagnostic performance. When ionizing radiation imaging cannot be avoided, cross-sectional imaging techniques are recommended instead of projection radiographies and by administering intravenous and peroral iodinated when indicated. Apart from mammography and radiographies of extremity sarcomas, projection radiography particularly chest radiography - adds little to cancer staging during pregnancy. Its low sensitivity in detecting metastases and low probability to alter treatment does not justify its radiation exposure. ${ }^{7}$

Importantly, the fetus only suffers risk from direct exposure within the field of examination and indirect fetal radiation doses resulting from internal scatter radiation are negligible. ${ }^{7}$ Therefore, as per American College of Radiology appropriateness criteria, abdominal shielding during radiography or CT procedures cannot substantially reduce fetal radiation and should never be performed. ${ }^{71415}$ Moreover, one should avoid wrapping a lead apron around the pelvis to shield the fetus directly exposed to radiation during abdominalpelvic $\mathrm{CT}$. The introduction of a highly attenuating material into the imaging area forces the CT-scanners radiation and imaging optimization system to drastically increase the tube radiation output in trying to penetrate the shield and maintain diagnostic image quality. ${ }^{14}$ This results in an increased dose to the fetus.

CT is a routine clinical modality in cancer imaging and adds value for the pregnant cancer patient. As mentioned before, radiation exposure to the fetus is negligible when outside the radiation field allowing safe performance of CT of the head, cervical spine, extremities (excluding the pelvis and hips), and thorax. ${ }^{79}$ The widespread and direct availability makes CT an attractive modality in case of emergencies. In the case of suspected pulmonary embolism, dedicated contrast-CT of the chest is highly accurate and safe with a lower fetal radiation exposure dose to the fetus compared with ventilation-perfusion scanning. ${ }^{16}$ For imaging the abdomen, with direct exposure to the fetus, the risks and benefits should be carefully weighted. Radiation dose exposure may be reduced by technical optimizations, including decrease of voltage and current, increase of pitch, widening of the beam collimation, and limiting the coverage of the scanned areas. ${ }^{17}$ Additionally, iterative reconstruction enables for adequate diagnostic accuracy while performing ultra-low-dose $\mathrm{CT} .^{18}$ 
The scan should be limited to a single contrast-phase scan in order to avoid cumulative radiation doses exceeding the $100 \mathrm{mGy}$ threshold. It is recommended to administer intravenous iodinated contrast as it significantly improves the information required for therapeutic management compared with non-enhanced $\mathrm{CT}^{7}$ Current standard radiological practice uses low-osmolarity, watersoluble iodinated contrast agents and, to date, no cases of neonatal hypothyroidism have been reported following administration during pregnancy. ${ }^{19}$ In countries with routine screening for neonatal hypothyroidism, no further action should be taken after birth. When no routine screening is available, a single laboratory test should be done in the first week after birth. ${ }^{79}$ Nevertheless, in the absence of contra-indications, MRI is preferred for imaging the abdomen and pelvis omitting any radiation exposure and allowing higher accuracy for characterization and local staging of pelvic tumors, nodal, hepatic, and peritoneal staging compared with $\mathrm{CT} .{ }^{11}$

Over the past years, PET imaging is increasingly being used next to bone scintigraphy for staging and response evaluation. ${ }^{11}$ Hybrid systems, combining nuclear imaging devices with $\mathrm{CT}$ are now the standard clinical routine. The two most commonly used tracers, $18 \mathrm{~F}$ fluorodeoxyglucose (FDG) and technetium $99 \mathrm{~m}$ may be used safely in pregnant patients. lodine-131 cannot be used safely during pregnancy as it can affect the fetal thyroid and should be replaced by technetium $99 \mathrm{~m}$ when essential for imaging the maternal thyroid. ${ }^{20}$ Radio-isotopes irradiate the fetus through accumulation in the maternal body tissues and organs, particularly the bladder, as well as by crossing the placental barrier and entering the fetal circulation. The amount of fetal radiation exposure depends on the weight of the fetus, the type of radiotracer, and the administered dose..$^{20}$

A number of precautions minimize fetal radiation dose during nuclear imaging. Dose calculation has to take into account physiological pregnancy changes that can alter the effective dose of different radiotracers: for intravenously injected tracers with renal excretion, a bladder catheter is placed simultaneously to intravenous hydration to avoid the accumulation of tracer in the maternal bladder. ${ }^{11}$ While the fetal radiation exposure to nuclear imaging tracers is relatively low, the now standard use of hybrid imaging with $\mathrm{CT}$ increases the final cumulative dose due to the combined CT and radio-isotope exposure. ${ }^{9}$ Therefore, PET/CT is not considered a primary imaging modality during pregnancy.

MRI is generally considered the preferred cross-sectional diagnostic modality and may be used regardless of gestational age. Concerns regarding the safety profile of MRI toward the fetus have been largely refuted and included potential teratogenic effects resulting from the static magnetic field, heating effects from radiofrequency resulting in fetal growth restriction, and acoustic noise from rapid gradient switching leading to hearing impairment. To date, no studies have unequivocally shown teratogenic effects to the fetus due to the magnetic field with a follow-up extending up to 9 years after the in-utero exposure. ${ }^{21}$ Post-natal hearing impairment or low birth weight have never been observed following MRI exposure and there is no evidence of actual harm of tissue heating at currently used field-strengths and clinical scan times. ${ }^{22}$

Nevertheless, a few specific and simple precautions minimize tissue heating. Radiofrequency pulses deposit energy as heat in the tissue measured as the specific absorption rate. Clinical MRI systems operate largely within the safety limits margin of $4 \mathrm{~W} /$ $\mathrm{kg}$ specific absorption rate (imposed by the FDA) while routinely available hardware and software components including multichannel phased-array coils and parallel transmission further minimize specific absorption rate. ${ }^{11}$

In pregnant patients, MRI systems are set to operate at a reduced setting of $2 \mathrm{~W} / \mathrm{kg}$ specific absorption rate. The MRI protocol is adapted to interleave low- and high-specific absorption rate sequences and limit the duration of the examination to a maximum of $30 \mathrm{~min}$. When the protocol adheres to these predefined settings, MRI can be performed up to 3 Tesla in every trimester of pregnancy. ${ }^{23}$ Contrary to this, the American College of Radiology advises extreme caution in using gadolinium as its effect on the fetus in terms of teratogenicity and risk of development of nephrogenic systemic fibrosis is unkown. ${ }^{23}$ Gadolinium chelates cross the placenta and circulate between the fetus and amniotic fluid which increases the probability of dechelation with long term exposure of the mother and fetus to free toxic gadolinium ions. It is therefore recommended to rely on non-contrast dependent sequences as much as possible. By combining high contrast anatomical sequences with the clinically available non-contrast dependent diffusion-weighted imaging (DWI) sequence, which allows functional characterization of tissue, this can nearly always be achieved. ${ }^{3}$

\section{GENERAL CONSIDERATIONS IN DIAGNOSTIC IMAGING IN PREGNANCY}

Ultrasound excels in providing diagnostic answers to definite questions concerning specific organs. It is routinely used for first-line locoregional assessment of breast cancer, thyroid cancer, and ovarian masses, as well as characterization of superficial lymph nodes. However, ultrasound has difficulties and is less reproducible to assess deeper abdominal structures and larger organs which can be aggravated by superimposing bowel gasses, air-filled lungs, obesity, and the pregnant uterus. Therefore, ultrasound should not be considered, when clinical evaluation warrants extensive staging.

MRI allows more reproducible evaluation of organ systems and - more recently - whole-body assessment. Additionally, MRI has excellent soft tissue contrast enabling highly detailed assessment of locoregional tumor extent with the added ability to combine highly detailed anatomical data with evaluation of functional tissue properties by DWI. This enables tumor detection, characterization, and response assessment by probing differential water molecule displacements based on cellular microstructure differences, which are quantified by (changes in) apparent diffusion coefficient. As such, DWI depicts tumorous lesions as bright lesions at high b-value of $1000 \mathrm{~s} / \mathrm{mm}^{2}$ in contrast to the suppressed background signal of organs, blood vessels, body fluids, and treatment- induced necrosis and fibrosis (Figure 1). Importantly, DWI does not need gadolinium to generate image contrast enhancing the safety profile of MRI for use in pregnant patients while reaching a reported accuracy for detecting primary tumors, nodal and distant metastases at least similar to gadolinium-enhanced MRI. ${ }^{24}$

CT remains the clinical standard for assessing lung metastases due to its high sensitivity and - given its minimal fetal radiation exposure - can safely replace chest radiographs. Chest CT should be used as upfront modality in the case of clinical symptoms indicating metastases or in the staging of tumors with a high propensity 

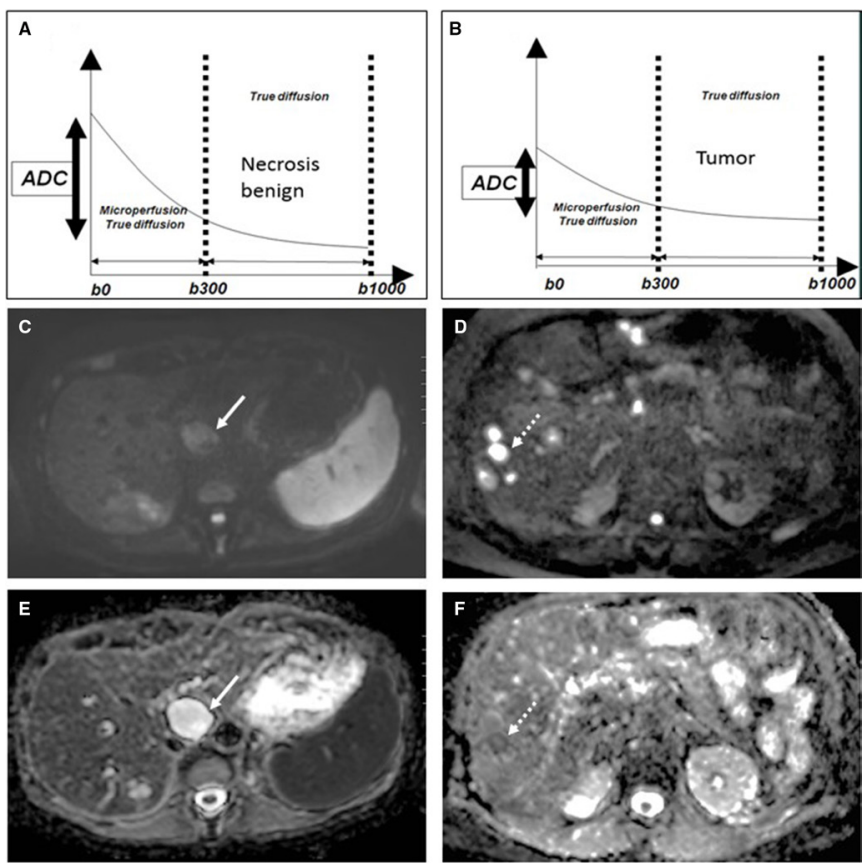

Figure 1 Diffusion-weighted imaging-based characterization of $(A, C, E)$ benign biliary cyst (arrow) versus $(B, D, F)$ malignant liver metastases (dashed arrow). (A) the signal decay curve of the biliary cyst shows strong decrease in signal intensity over the consecutive b-value sequences resulting in high calculated apparent diffusion coefficient. (C) on the b1000 image. The biliary cyst appears with low signal intensity and (E) white on the apparent diffusion coefficient map. (B) the signal decay curve of the liver metastasis shows only a limited decrease in signal intensity over the consecutive b-value sequences, resulting in a low calculated apparent diffusion coefficient. (D) on the b1000 image, the liver metastasis appears bright and (F) dark on the apparent diffusion coefficient map.

of developing lung metastases including sarcomas, head and neck cancers, and melanoma. ${ }^{7}$

There is increasing evidence that MRI may show similar performance to CT for detecting lung metastases. ${ }^{25}$ This may reduce the need for chest CT, particularly when staging is performed with whole body diffusion-weighted/MRI. Due to the relative rarity and diversity of cancers during pregnancy, it is difficult to develop standardized imaging protocols and as a disadvantage, this approach requires multimodality planning. Therefore, efforts are made for the clinical integration of single-step imaging modalities to stage advanced cancer with absent or reduced radiation dose by using whole-body diffusion-weighted/MRI and hybrid PET/MRI. Recent studies have demonstrated excellent diagnostic performance of whole-body diffusion-weighted/MRI for detecting distant metastases, lymph node metastases, and lymphoma superior to CT and at least equivalent to FDG-PET/CT ${ }^{24}$ Whole-body diffusion-weighted/ MRI has been evaluated in the specific setting of pregnant patients with cancer showing very good interreader agreement, accuracy of $90 \%$ for primary tumor detection, accuracy between $98.5 \%$ and $99.5 \%$ for detecting nodal metastases, and accuracy between $90 \%$ and $100 \%$ for detecting distant metastases. ${ }^{24}{ }^{26}$ Whole-body diffusion-weighted/MRI has shown particularly high diagnostic value for assessing hepatic and peritoneal metastases as well as skeletal metastases compared with contrast-enhanced MRI, contrast-enhanced CT, or FDG-PET/CT and bone scintigraphy, respectively. ${ }^{27}$ Hybrid PET/MRI allows for a reduction in dose exposure to the fetus with reported better detection of brain, bone, and liver metastases compared with PET/CT. DWI adds to the sensitivity while PET adds specificity. However, so far, PET/MRI has not been specifically investigated in a systematic series of pregnant cancer patients. $^{28}$

Currently, clinical whole-body diffusion-weighted/MRI and PET/ MRI are mostly performed in large-volume expert centers. The widespread availability of MR systems and required sequences, clinically efficient scan time of on average $38 \mathrm{~min}$, increasing standardization in imaging technique and interpretation, increasing radiologist training, and complete absence of radiation is likely to facilitate the more widespread use of whole-body diffusionweighted/MRI for staging advanced cancers during pregnancy. ${ }^{29}$ Appropriate diagnostic work-up should result in an accuracy equal to non-pregnant patients, without fetal risk, using only the imaging modalities according to the need for assessment of locoregional disease extent and probability of distant staging. The diagnostic pathway is personalized to the patient through a prestaging multidisciplinary board discussion in accordance with local imaging expertize, by tumor type and available imaging equipment. ${ }^{11}$

\section{DIAGNOSIS AND STAGING BY TUMOR TYPE}

\section{Breast cancer}

Breast cancer during pregnancy presents mostly as a palpable mass, and its finding should warrant immediate investigation. ${ }^{4}$ Breast ultrasound is the first-line imaging modality (Figure 2). It allows immediate identification of obvious benign lesions such as cysts and galactocoeles that require no further evaluation and has shown an overall pooled sensitivity and specificity of $80.1 \%$ and $88.4 \%$, respectively, for detection of malignancy and any solid
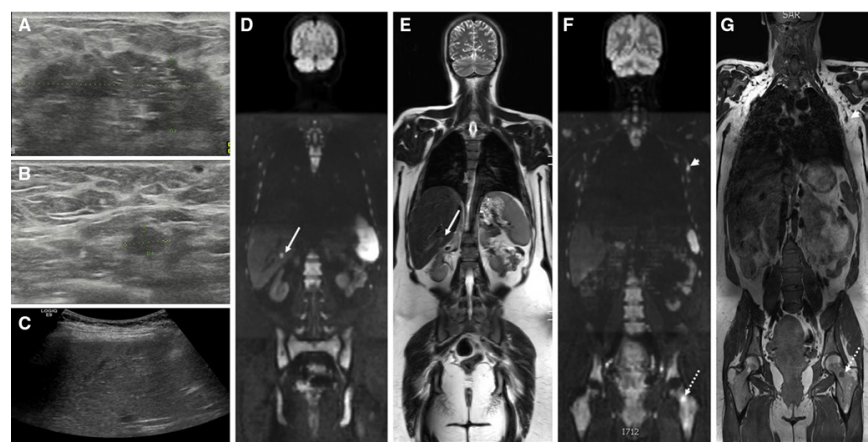

Figure 2 Pregnant patient with locally advanced breast cancer: breast ultrasound shows (A) large tumorous mass in the left breast with $(B)$ a regional lymphadenopathy in the left axilla. (C) screening ultrasound is equivocal. at whole body diffusion-weighted MRI, (D) the b1000 diffusion-weighted sequence shows a bright lesion in the liver, corresponding to a solid lesion at (E) T2-weighted sequence compatible with liver metastasis (arrows). Additionally, (F) the b1000 diffusionweighted sequence confirms the lymph node metastasis (arrowheads) as a bright lesion and nodular shape at (G) T1weighted sequence and shows a bright lesion corresponding to T1 hypo-intensity in the left femur compatible with bone metastasis (dashed arrows). 
breast lesion warrants a biopsy. ${ }^{30}$ Mammography is additionally used by acquiring one mediolateral oblique view to look for microcalcifications or tissue distortion supporting the diagnosis of malignancy, in case of equivocal ultrasound. ${ }^{731}$

In case of malignancy, locoregional and distant staging is required for further treatment planning and comprises the assessment of primary tumor properties, including tumor size, local extent, multifocality in the ipsi- or contralateral breast, and assessment of regional and supraclavicular lymph nodes. For this purpose, ultrasound is combined with mammography with craniocaudal and mediolateral oblique views of both breasts in order to aid in delineation of tumor extent and multifocality when presenting with microcalcifications. ${ }^{731}$ This combined approach has an incremental cancer detection rate of $15.5 \%$ in the ipsilateral breast and $3.9 \%$ in the contralateral breast, and has substantial impact on surgical management. ${ }^{32}$ As the incremental cancer detection rate of ultrasound combined with mammography is similar to that of breast MRI, it allows to omit the MRI from the diagnostic work-up in pregnant patients, thereby avoiding exposure of the fetus to gadolinium.

Ultrasound is also the modality of choice for pretreatment regional staging and assessment of possible supraclavicular nodal metastases. In addition to prognostic information, it helps the physician in deciding between sentinel node biopsy, upfront axillar lymph node dissection, or neo-adjuvant treatment. Reported sensitivities and specificities for ultrasound vary between $26.4 \%$ and $92 \%$ with specificities of $55.6 \%-98.1 \%{ }^{33}$ Suspicious lymph nodes at ultrasound should be confirmed by percutaneous procedures, either fine needle aspiration cytology or core needle biopsy.

The imaging modalities used for distant staging depends on tumor stage. The lungs, liver, and skeleton are the most frequently affected sites. Asymptomatic women with early-stage breast cancer are generally assessed by chest radiography - although its diagnostic value can be questioned - and liver ultrasound. Advanced imaging should be reserved for patients with symptoms suspicious for metastases, (node-positive) locally advanced breast cancer, and patients with an elevated tumor marker. In this setting, staging may be performed by chest CT combined with MRI with DWI sequence for assessment of the liver due to its high sensitivity of on average $87.1 \%$ for detecting liver metastases. ${ }^{34}$ For detecting bone metastases, an MRI with T1-weighted sequence and DWI of at least the full-spine and pelvis is the preferred imaging modality. Bone scintigraphy is only recommended in the case of unavailability of or unequivocal findings at MRI, although its value during pregnancy is unclear. ${ }^{11}$

When available, whole body diffusion-weighted/MRI can be used for single-step staging (Figure 2). ${ }^{24} 26$ It allows better detection of skeletal metastases than $\mathrm{CT}$ and bone scintigraphy with equal diagnostic performance as PET/CT, as well as sensitive detection liver and peritoneal metasases. ${ }^{27}$ For detecting lung metastases, DWI needs correlation with breath-hold Dixon-based anatomical sequences in the MR-protocol to be sufficiently sensitive. ${ }^{25}$ The DWI sequence may make the whole-body diffusion-weighted/ MRI sequence of complementary value to ultrasound for regional staging of the breast. DWI shows sensitivities between $72.4 \%$ and $97 \%$ with specificities between $54.4 \%$ and $91.7 \%$ for regional nodal staging in breast cancer and reported sensitivity between $75.7 \%$ and $78.9 \%$ to assess multifocality or contralateral breast involvement. ${ }^{3524}$
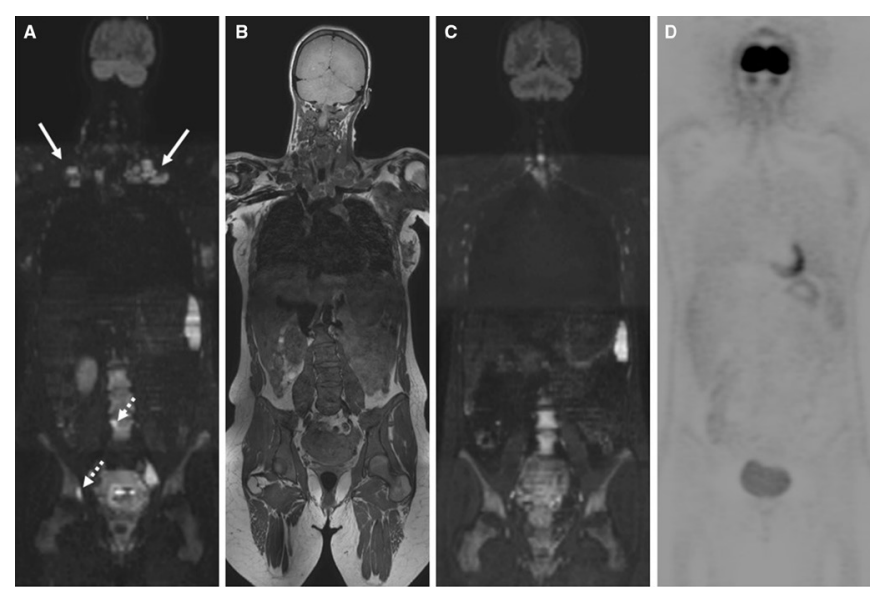

Figure 3 Incidental finding of Hodgkin lymphoma after suspect non-invasive prenatal testing in pregnant patient: at whole-body diffusion-weighted MRI, (A) the b1000 diffusion-weighted sequence shows bright and enlarged lymphadenopathies in the bilateral base of neck (arrows) and two bright lesions of bone marrow involvement located at the right acetabulum and the fourth lumbar vertebral body corresponding to diffuse hypo-intensity at $(B)$ the T1-weighted sequence. (C) interim whole-body diffusionweighted MRI after two cycles of chemotherapy shows complete response. (D) complete response is confirmed at end of treatment hybrid positron emission tomography/CT after delivery.

\section{Lymphoma}

Hodgkin lymphoma is the most frequent hematological tumor during pregnancy, given its tendency to occur in women in the reproductive age. ${ }^{1}$ There is debate regarding the therapeutic management and the subsequent requirement for advanced staging as Hodgkin lymphoma has a slow growing nature for which treatment could be deferred until after delivery. However, symptomatic and advancedstage Hodgkin lymphoma might require immediate treatment and correct staging aids in maternal clinical management, impacts long-term survival, and influences fetal management as standard chemotherapy may affect fetal growth, requiring regular obstetric follow-up. ${ }^{36}$

$\mathrm{PET} / \mathrm{CT}$ is the clinical standard in lymphoma, but whole-body diffusion-weighted/MRI has shown excellent concordance with up to $99.4 \%$ with FDG-PET/CT for detecting nodal and extranodal lymphoma, including an accuracy to determine bone marrow involvement up to $93 \%{ }^{27} 2937$ Staging by PET/CT or whole- body diffusion-weighted/MRI leads to similar progression-free survival curves indicating that both techniques have a similar impact on patient management. ${ }^{38}$ Thus, in pregnant patients with lymphoma, whole-body diffusion-weighted/MRI may be considered a nonirradiating alternative to FDG-PET/CT for diagnosing and staging lymphoma (Figure 3). Importantly, whole-body diffusion-weighted/ MRI allows reliable interim and post-treatment response evaluation with reported agreement of over 99.2 with PET/CT for predicting outcome. ${ }^{37}$ This is of particular importance in Hodgkin lymphoma, where interim response assessment may help to predict outcome and tailor treatment to decrease maternal and fetal toxicity (Figure 3). ${ }^{36}$ 


\section{Review}

\section{Cervical cancer}

For cervical cancer, treatment strategies differ depending on the stage of pregnancy at time of diagnosis and clinical tumor stage. Similar to non-pregnant patients, MRI is established as a complementary diagnostic tool to clinical examination for locoregional staging. As an advantage, MRI does not depend on gadoliniumcontrast to assess locoregional disease extent and allows accurate and reproducible assessment of prognostic tumor properties including tumor size, parametrial and pelvic wall invasion, adjacent organ invasion, lymph nodes, and metastases. ${ }^{34} 27$

By combining the T2-weighted sequence with DWI (T2/DWI), MRI shows highest accuracy for locoregional staging. In one study, T2/DWI improved specificity for detecting parametrial invasion over T2-weighted MRI alone from $85.2 \%-88.7 \%$ to $96.5 \%-99 \%$ with unchanged sensitivity between $67 \%$ and $75 \%$, resulting in an improved positive predictive value and reader confidence. 3940 In another study, T2/DWI improved sensitivity over T2-weighted MRI alone from $86 \%$ to $90 \%$ for predicting parametrial invasion. ${ }^{15}$ DWI also adds accuracy for nodal staging, including subcentimeter lymph nodes. In a meta-analysis pooling 67 studies, the area under the curve for FDG-PET/CT (0.92) and DWI (0.90) for nodal staging were significantly better than for CT $(0.83)$. DWI shows highest sensitivity of $88 \%$ for detecting lymph node metastases but with moderate specificity of $83 \% .{ }^{41} \mathrm{DWI}$ can thus provide complementary information but not avoid planned surgical staging in pregnant patients with cervical cancer.

Additionally, MRI is a reproducible modality to assess response to neo-adjuvant chemotherapy. ${ }^{3}$ For response evaluation after neoadjuvant chemotherapy for stage IB1 to IIB cervical cancers, MRI and transrectal ultrasound show similar accuracy of $77 \%$ for the detection of residual tumor but with highest sensitivity for MRI (96\%). ${ }^{42}$

To achieve the highest possible accuracy, the MRI protocol for locoregional staging of cervical cancer during pregnancy and response assessment should combine T2/DWI MRI and cover the entire abdomen in order to inform on local tumor extension, pelvicaortic lymph nodes, liver, bone, and peritoneum. Additional contrastenhanced chest CT is indicated to assess thoracic metastases
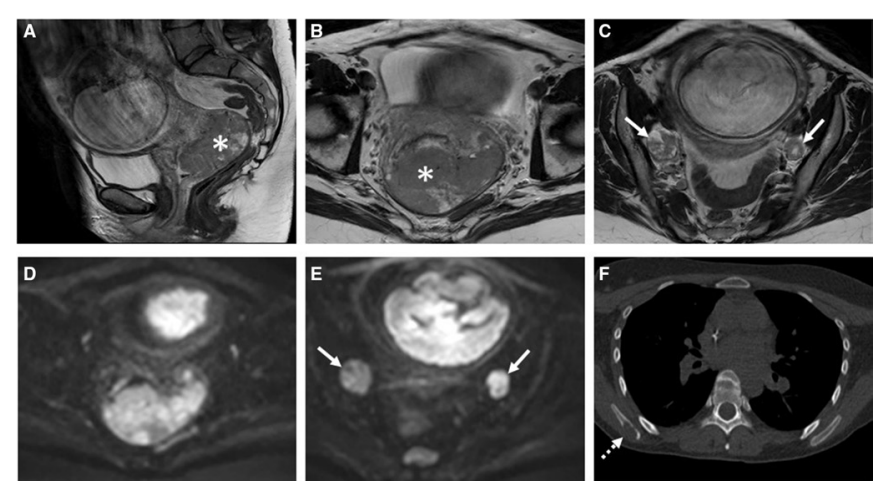

Figure 4 Pregnant patient with diagnosis of uterine cervical cancer. (A-C) T2-weighted MRI combined with $(D, E)$ diffusion-weighted imaging of the pelvis shows a large tumorous mass (asterisk) in the cervix and prolabating in the proximal vagina without parametrial invasion but with bilateral para-iliac lymphadenopathies (arrows). (F) staging chest CT shows an osteolytic lesion in the right scapula compatible with skeletal metastasis.
(Figure 4). An FDG-PET with low-dose CT can be applied in case of equivocal distant findings critical for curative treatment. ${ }^{4}$

\section{Ovarian cancer}

Transvaginal ultrasound is the primary imaging modality to assess ovarian cysts during pregnancy. The International Ovarian Tumor Analysis studies have established rules and models to characterize ovarian pathology by examiners with different levels of ultrasound expertize and have validated subjective criteria, rules, risk models, and combined interpretation strategies with sensitivity up to $90 \%-96 \%$ for characterizing ovarian cysts. ${ }^{42}$ MRI shows equally high accuracy as transvaginal ultrasound for characterizing ovarian masses, with potential value to assess the sonographically indeterminate mass, but relies heavily on dynamic contrast-enhanced MRI for the prediction of malignancy additional to DWI and standard T1-weighted and T2-weighted characteristics. ${ }^{43}$ Omitting dynamic contrast-enhanced MRI from the protocol - as required in pregnancy - will inevitably result in lower specificity for predicting malignancy. In contrast, the prediction of benign features depends mostly on the non-contrast sequences. Therefore, during pregnancy, pelvic MRI is probably most reliable in ruling out malignancy in an ovarian mass. ${ }^{43}$

When advanced stage ovarian cancer is diagnosed, neoadjuvant chemotherapy until fetal maturation and complete cytoreduction after delivery is the favored treatment option. ${ }^{4}$ In several studies, whole-body diffusion-weighted/MRI allowed prediction of the primary tumor (primary ovarian cancer vs other origin), and peritoneal disease load with higher accuracy than CT and PET/CT, and detected distant metastases with equal diagnostic performance as PET/CT. ${ }^{44}$ DWI assessment of peritoneal metastases correlates well with surgical peritoneal load and whole-body diffusionweighted/MRI has shown to be significantly more accurate than CT for predicting operability and detection of unresectable tumor sites (Figure 5). ${ }^{45}$ In our experience, omitting gadolinium during pregnancy does not significantly reduce the diagnostic capability of whole-body diffusion-weighted/MRI as it relies mainly on DWI for disease detection. Whole-body diffusion-weighted/MRI can be considered a highly valuable diagnostic modality in staging and operability assessment of the non-pregnant and pregnant patient with ovarian cancer.

\section{Gastrointestinal cancer}

During pregnancy, colorectal tumors are the most common gastrointestinal cancers. Depending on disease stage, treatment consists of chemotherapy and surgery. The most common sites of metastases are lymph nodes, liver, lung, and peritoneum. CT is most commonly used for staging the thorax and abdomen in cancer patients. However, in pregnant patients, ultrasound and/or (mainly) MRI are appropriate alternatives. Ultrasound helps in initial screening of liver metastases but in current clinical practice, treatment for colorectal cancer is only initiated after staging by crosssectional imaging, irrespective of tumor stage.

$\mathrm{MRI}$ is the imaging modality of choice for locoregional staging of rectal cancer, allowing accurate assessment of the primary tumor, lymph nodes, the mesorectal fascia, and the presence of extramural venous invasion. Furthermore, MRI is the preferred first-line imaging study for evaluating colorectal liver metastases in patients who have not previously undergone therapy, especially for those 

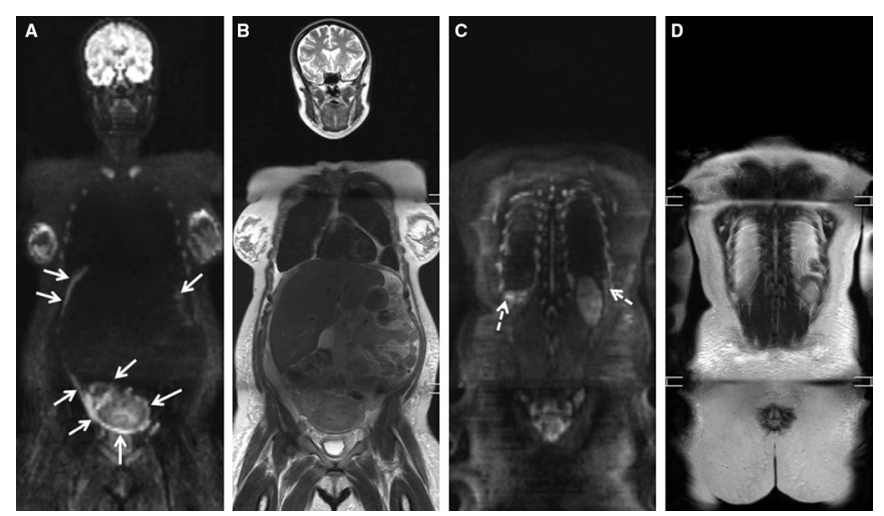

Figure 5 Pregnant patient diagnosed with a malignant ovarian mass at gynecological ultrasound. $(A, C) B 1000$ whole-body diffusion-weighted MRI shows multifocal and diffuse bright confluent peritoneal and pleural lesions compatible with diffuse metastases. correlative. (B,D) T2weighted images show thickening of the peritoneal planes, ascites respectively bilateral pleural fluid.

patients who will potentially undergo hepatic resection. ${ }^{46}$ MRI may also be used as a complete one-time imaging method for colorectal cancer patients, providing staging information of the entire body. The whole-body diffusion-weighted/MRI has shown equivalent diagnostic value as to standard diagnostic pathways using CT for the staging of colorectal cancer and reduces the number of tests needed. ${ }^{47}$ Similar to ovarian cancer, whole-body diffusionweighted/MRI allows better detection of peritoneal metastases than CT with $97.8 \%$ sensitivity compared with $43.2 \%$. Moreover, wholebody diffusion-weighted/MRI allows significantly better prediction of operability compared with CT with $90.6 \%$ sensitivity compared with $25 \% .{ }^{48}$ PET scans with or without integrated CT do not appear to add significant information to CT scans alone for routine preoperative staging of colorectal cancer. ${ }^{49}$ Based on these data, wholebody diffusion-weighted/MRI - or alternatively - MR abdomen with chest CT are safe and accurate alternatives to standard CT and $\mathrm{PET} / \mathrm{CT}$ for the staging of colon cancer during pregnancy.

\section{Thyroid cancer}

Optimal treatment of thyroid cancer depends on accurate staging of the extent of disease at presentation. Combined ultrasound and fine needle aspiration cytology are the primary modalities to diagnose thyroid cancer and assess regional lymph nodes. ${ }^{50}$ Next to distinct anatomical features at ultrasound comparable to other head and neck cancers, nodal metastases from thyroid cancer are characterized by hypervascularity and are easily depicted by color-doppler. Ultrasound is superior to CT or MRI for regional nodal staging, with a reported accuracy of $89 \%$ at a minimum nodal size of $6 \mathrm{~mm}$.

Small thyroid cancers confined to the gland (T1-T2), receive no further imaging in addition to ultrasound. Larger tumors (T3-T4) require additional assessment of local disease extent for surgical guidance. For this purpose, non-contrast enhanced MRI with DWI is preferred over CT to avoid the use of iodinated contrast which can interfere with lodine-131 for diagnostic whole-body scintigraphy and treatment after birth. ${ }^{50}$ Preferred sites for distant metastases include the lungs, bone, and, to lesser extent, the liver or other sites. ${ }^{7}$ In general, pre-operative distant staging is only performed in case of anaplastic thyroid cancer or symptoms indicative of metastases.
Chest CT is the preferred modality to screen for lung metastases. Imaging of other sites including the liver and bone are primarily assessed by MRI with DWI similar to breast cancer. Well differentiated (papillary and follicular) thyroid cancers do not undergo preoperative distant staging as distant metastases, particularly to the lungs, still have relatively good prognosis and do not impact on the decision to perform thyroidectomy and neck dissection. ${ }^{51}$ Definitive staging is mostly done in the post-operative phase - and for pregnant patients after delivery - with a lodine- 131 whole-body scintigraphy during ablative iodine therapy. ${ }^{7951}$ Additional chest CT may be performed to identify miliary metastases.

\section{Melanoma}

Imaging staging in melanoma is guided by the depth of invasion and regional lymph node status, as these are the most important prognostic factors. ${ }^{52}$ Ultrasound is used as the primary imaging modality at the time of diagnosis to assess regional lymph node involvement with reported sensitivities between $39 \%$ and $89.4 \%$. Accuracy may be increased by adding fine needle aspiration cytology or core needle biopsy. ${ }^{53}$ However, as no imaging modality can exclude nodal micrometastases, sentinel node biopsy is the standard in clinically node-negative melanoma patients and is safe in pregnant patients. ${ }^{54}$

In patients with low risk for distant metastases, chest radiography has no value and should be replaced by chest CT if warranted. Liver and regional lymph node are assessed with ultrasound. In patients with high risk of metastases, evaluation of distant metastases may be performed by combining non-contrast MRI of the brain, chest CT, and MRI including DWI of the liver and axial skeleton. An FDG-PET with low-dose CT may be considered in case of equivocal distant findings critical for curative treatment. Whole-body diffusionweighted/MRI shows higher sensitivity for detecting liver, bone, and brain metastases than PET/CT, be it with lower accuracy for detecting nodal metastases and represents a valid alternative for staging high-risk patients during pregnancy. ${ }^{27} 55$

\section{CONCLUSION}

Imaging of pregnant cancer patients is challenging. Appropriate diagnostic work-up should result in an accuracy equal to nonpregnant patients, without fetal risk, using only the imaging modalities sufficient to allow modification of treatment. The need to balance potential conflicts between maternal benefit and fetal risk makes it difficult to standardize the diagnostic approach and harbors the risk of underutilizing diagnostic imaging with suboptimal diagnostic (and consequently therapeutic) management as compared with non-pregnant patients.

Therefore, a multidisciplinary team, including radiologists and nuclear imaging physicians should decide on optimal diagnostic strategies.

Due to their lack of ionizing radiation and highly accurate clinical performance, ultrasound and MRI are the preferred primary imaging modalities, while ionizing radiation imaging modalities including CT, PET/CT and bone scintigraphy can be selectively performed in unresolved cases when the benefit for the mother clearly outweighs the risk to the fetus and when taking care not to exceed $100 \mathrm{mGy}$ of fetal exposure. Whole-body diffusion-weighted/MRI and PET/MRI 


\section{Review}

hold promise for accurate single-step staging with the absence or reduction of fetal radiation.

\section{Author affiliations}

${ }^{1}$ Department of Radiology, University Hospitals Leuven, Leuven, Belgium

${ }^{2}$ Department of Imaging \& Pathology, KU Leuven, Leuven, Belgium

${ }^{3}$ Department of Gynaecology and Obstetrics, University Hospitals Leuven, Leuven, Belgium

${ }^{4}$ Department of Oncology, KU Leuven, Leuven, Belgium

${ }^{5}$ Center for Gynecological Oncology, Academic Medical Centre AmsterdamUniversity of Amsterdam and The Netherlands Cancer Institute-Antoni van Leeuwenhoek Hospital, Amsterdam, The Netherlands

${ }^{6}$ Department of Radiology, Cliniques Universitaires Saint-Luc, Institut de Recherche Expérimentale et Clinique (IREC), Université Catholique de Louvain (UCLouvain), Brussels, Belgium

${ }^{7}$ Department of Development and Regeneration, KU Leuven, Leuven, Belgium

Contributors All authors contributed to the following (adherent to ICMJE standards): substantial contributions to the conception or design of the work; or the acquisition, analysis, or interpretation of data for the work; drafting the work or revising it critically for important intellectual content; final approval of the version to be published; and agreement to be accountable for all aspects of the work in ensuring that questions related to the accuracy or integrity of any part of the work are appropriately investigated and resolved.

Funding The authors have not declared a specific grant for this research from any funding agency in the public, commercial, or not-for-profit sectors.

Competing interests None declared.

Patient consent for publication Not required.

Provenance and peer review Not commissioned; externally peer reviewed.

Open access This is an open access article distributed in accordance with the Creative Commons Attribution Non Commercial (CC BY-NC 4.0) license, which permits others to distribute, remix, adapt, build upon this work non-commercially, and license their derivative works on different terms, provided the original work is properly cited, an indication of whether changes were made, and the use is noncommercial. See: http://creativecommons.org/licenses/by-nc/4.0/.

\section{ORCID iD}

Vincent Vandecaveye http://orcid.org/0000-0002-0800-3279

\section{REFERENCES}

1 de Haan J, Verheecke M, Van Calsteren K, et al. Oncological management and obstetric and neonatal outcomes for women diagnosed with cancer during pregnancy: a 20-year international cohort study of 1170 patients. Lancet Oncol 2018;19:337-46.

2 Amant F, Verheecke M, Wlodarska I, et al. Presymptomatic identification of cancers in pregnant women during noninvasive prenatal testing. JAMA Oncol 2015;1:814-9.

3 Gui B, Cambi F, Micco M, et al. MRI in pregnant patients with suspected abdominal and pelvic cancer: a practical guide for radiologists. Diagn Interv Radiol 2020;26:183-92.

4 Amant F, Halaska MJ, Fumagalli M, et al. Gynecologic cancers in pregnancy: guidelines of a second international consensus meeting. Int J Gynecol Cancer 2014;24:394-403.

5 Benn P, Plon SE, Bianchi DW. Current controversies in prenatal diagnosis 2: NIPT results suggesting maternal cancer should always be disclosed. Prenat Diagn 2019;39:339-43.

6 Amant F, Vandenbroucke T, Verheecke M, et al. Pediatric outcome after maternal cancer diagnosed during pregnancy. $N$ Engl J Med 2015;373:1824-34.

7 Woitek R, Prayer D, Hojreh A, et al. Radiological staging in pregnant patients with cancer. ESMO Open 2016;1:e000017.

8 O'Brien WD, Deng CX, Harris GR, et al. The risk of exposure to diagnostic ultrasound in postnatal subjects: thermal effects. $J$ Ultrasound Med 2008;27:517-35.

9 American College of Obstetricians and Gynecologists' Committee on Obstetric Practice. Committee opinion no. 656: guidelines for diagnostic imaging during pregnancy and lactation. Obstet Gynecol 2016;127:e75-80.

10 Torloni MR, Vedmedovska N, Merialdi M, et al. Safety of ultrasonography in pregnancy: WHO systematic review of the literature and meta-analysis. Ultrasound Obstet Gynecol 2009;33:599-608.

11 de Haan J, Vandecaveye V, Han SN, et al. Difficulties with diagnosis of malignancies in pregnancy. Best Pract Res Clin Obstet Gynaecol 2016;33:19-32

12 Gjelsteen AC, Ching BH, Meyermann MW, et al. CT, MRI, PET, PET/ $\mathrm{CT}$, and ultrasound in the evaluation of obstetric and gynecologic patients. Surg Clin North Am 2008;88:361-90.

13 Tirada N, Dreizin D, Khati NJ, et al. Imaging pregnant and lactating patients. Radiographics 2015;35:1751-65.

14 Marsh RM, Silosky M. Patient shielding in diagnostic imaging: discontinuing a legacy practice. AJR Am J Roentgenol 2019;212:755-7.

15 ACR-SPR practice parameter for imaging pregnant or potentially pregnant adolescents and women with ionizing radiation. amended, 2014. Available: http://www.acr.org/ /media/9e2ed55531fc4b4fa53e f3b6d3b25df8.pdf

16 Patel SJ, Reede DL, Katz DS, et al. Imaging the pregnant patient for nonobstetric conditions: algorithms and radiation dose considerations. Radiographics 2007;27:1705-22.

17 Wang PI, Chong ST, Kielar AZ, et al. Imaging of pregnant and lactating patients: Part 1, evidence-based review and recommendations. AJR Am J Roentgenol 2012;198:778-84.

18 Prakash P, Kalra MK, Kambadakone AK, et al. Reducing abdominal CT radiation dose with adaptive statistical iterative reconstruction technique. Invest Radiol 2010;45:202-10.

19 Bourjeily G, Chalhoub M, Phornphutkul C, et al. Neonatal thyroid function: effect of a single exposure to iodinated contrast medium in utero. Radiology 2010;256:744-50.

20 Adam A, Dixon AK, Gillard JH, et al, eds. Grainger \& Allison's diagnostic radiology: a textbook of medical imaging. 6 th edn. New York (NY): Churchill Livingstone/Elsevier, 2015.

21 Bouyssi-Kobar M, du Plessis AJ, Robertson RL, et al. Fetal magnetic resonance imaging: exposure times and functional outcomes at preschool age. Pediatr Radiol 2015;45:1823-30.

22 Strizek B, Jani JC, Mucyo E, et al. Safety of MR imaging at $1.5 \mathrm{~T}$ in fetuses: a retrospective case-control study of birth weights and the effects of acoustic noise. Radiology 2015;275:530-7.

23 Expert Panel on MR Safety, Kanal E, Barkovich AJ, et al. ACR guidance document on MR safe practices: 2013. J Magn Reson Imaging 2013;37:501-30.

24 Han SN, Amant F, Michielsen K, et al. Feasibility of whole-body diffusion-weighted MRI for detection of primary tumour, nodal and distant metastases in women with cancer during pregnancy: a pilot study. Eur Radiol 2018;28:1862-74.

25 Stolzmann P, Veit-Haibach P, Chuck N, et al. Detection rate, location, and size of pulmonary nodules in trimodality PET/CT-MR: comparison of low-dose CT and Dixon-based MR imaging. Invest Radiol 2013;48:241-6.

26 Peccatori FA, Codacci-Pisanelli G, Del Grande M, et al. Whole body MRI for systemic staging of breast cancer in pregnant women. Breast 2017;35:177-81.

27 Petralia G, Padhani AR, Pricolo P, et al. Whole-body magnetic resonance imaging (WB-MRI) in oncology: recommendations and key uses. Radiol Med 2019;124:218-33.

28 Partovi S, Kohan A, Rubbert C, et al. Clinical oncologic applications of PET/MRI: a new horizon. Am J Nucl Med Mol Imaging 2014;4:202-12.

29 Tunariu N, Blackledge M, Messiou C, et al. What's new for clinical whole-body MRI (WB-MRI) in the 21st century?. Br J Radiol 2020;93:20200562

30 Sood R, Rositch AF, Shakoor D, et al. Ultrasound for breast cancer detection globally: a systematic review and meta-analysis. J Glob Oncol 2019;5:1-17.

31 Taylor D, Lazberger J, Ives A, et al. Reducing delay in the diagnosis of pregnancy-associated breast cancer: how imaging can help us. $J$ Med Imaging Radiat Oncol 2011;55:33-42.

32 Candelaria RP, Huang ML, Adrada BE, et al. Incremental cancer detection of locoregional restaging with diagnostic mammography combined with whole-breast and regional nodal ultrasound in women with newly diagnosed breast cancer. Acad Radiol 2017;24:191-9.

33 Alvarez S, Añorbe E, Alcorta P, et al. Role of sonography in the diagnosis of axillary lymph node metastases in breast cancer: a systematic review. AJR Am J Roentgenol 2006;186:1342-8.

$34 \mathrm{Wu}$ L-M, Hu J, Gu H-Y, et al. Can diffusion-weighted magnetic resonance imaging (DW-MRI) alone be used as a reliable sequence for the preoperative detection and characterisation of hepatic metastases? A meta-analysis. Eur J Cancer 2013;49:572-84. 
35 Amornsiripanitch N, Bickelhaupt S, Shin HJ, et al. Diffusionweighted MRI for unenhanced breast cancer screening. Radiology 2019;293:504-20.

36 Maggen C, Dierickx D, Lugtenburg P, et al. Obstetric and maternal outcomes in patients diagnosed with Hodgkin lymphoma during pregnancy: a multicentre, retrospective, cohort study. Lancet Haematol 2019;6:e551-61.

37 Mayerhoefer ME, Karanikas G, Kletter K, et al. Evaluation of diffusion-weighted MRI for pretherapeutic assessment and staging of lymphoma: results of a prospective study in 140 patients. Clin Cancer Res 2014;20:2984-93.

38 Tsuji K, Kishi S, Tsuchida T, et al. Evaluation of staging and early response to chemotherapy with whole-body diffusion-weighted MR in malignant lymphoma patients: a comparison with FDG-PET/CT. J Magn Reson Imaging 2015;41:1601-7.

39 Park JJ, Kim CK, Park SY, et al. Parametrial invasion in cervical cancer: fused T2-weighted imaging and high-b-value diffusionweighted imaging with background body signal suppression at $3 \mathrm{~T}$. Radiology 2015;274:734-41.

40 Exner M, Kühn A, Stumpp P, et al. Value of diffusion-weighted MRI in diagnosis of uterine cervical cancer: a prospective study evaluating the benefits of DWI compared to conventional Mr sequences in a 3T environment. Acta Radiol 2016:57:869-77.

41 Liu B, Gao S, Li S. A comprehensive comparison of CT, MRI, positron emission tomography or positron emission tomography/CT, and diffusion weighted imaging-MRI for detecting the lymph nodes metastases in patients with cervical cancer: a meta-analysis based on 67 studies. Gynecol Obstet Invest 2017;82:209-22.

42 Andreotti RF, Timmerman D, Strachowski LM, et al. O-RADS us risk stratification and management system: a consensus guideline from the ACR ovarian-adnexal reporting and data system committee. Radiology 2020;294:168-85.

43 Thomassin-Naggara I, Poncelet E, Jalaguier-Coudray A, et al. Ovarian-adnexal reporting data system magnetic resonance imaging (O-RADS MRI) score for risk stratification of Sonographically indeterminate adnexal masses. JAMA Netw Open 2020;3:e1919896.

44 Vandecaveye V, Dresen R, De Keyzer F. Novel imaging techniques in gynaecological cancer. Curr Opin Oncol 2017;29:335-42.
45 Michielsen K, Dresen R, Vanslembrouck R, et al. Diagnostic value of whole body diffusion-weighted MRI compared to computed tomography for pre-operative assessment of patients suspected for ovarian cancer. Eur J Cancer 2017;83:88-98.

46 Niekel MC, Bipat S, Stoker J. Diagnostic imaging of colorectal liver metastases with CT, MR imaging, FDG PET, and/or FDG PET/CT: a meta-analysis of prospective studies including patients who have not previously undergone treatment. Radiology 2010;257:674-84.

47 Taylor SA, Mallett S, Beare S, et al. Diagnostic accuracy of wholebody MRI versus standard imaging pathways for metastatic disease in newly diagnosed colorectal cancer: the prospective streamline C trial. Lancet Gastroenterol Hepatol 2019;4:529-37.

48 Dresen RC, De Vuysere S, De Keyzer F, et al. Whole-Body diffusionweighted MRI for operability assessment in patients with colorectal cancer and peritoneal metastases. Cancer Imaging 2019;19:1-10.

49 Furukawa $\mathrm{H}$, Ikuma $\mathrm{H}$, Seki $\mathrm{A}$, et al. Positron emission tomography scanning is not superior to whole body multidetector helical computed tomography in the preoperative staging of colorectal cancer. Gut 2006;55:1007-11.

50 King AD. Imaging for staging and management of thyroid cancer. Cancer Imaging 2008;8:57-69.

51 Nixon IJ, Whitcher MM, Palmer FL, et al. The impact of distant metastases at presentation on prognosis in patients with differentiated carcinoma of the thyroid gland. Thyroid 2012;22:884-9.

52 Kauffmann RM, Chen SL. Workup and staging of malignant melanoma. Surg Clin North Am 2014;94:963-72. vii.

53 Ungureanu L, Botar Jid C, Candrea E, et al. The role of lymph node ultrasound evaluation in melanoma - review of the literature. Med Ultrason 2016;18:224-30.

54 Andtbacka RHI, Donaldson MR, Bowles TL, et al. Sentinel lymph node biopsy for melanoma in pregnant women. Ann Surg Oncol 2013;20:689-96.

55 Pfannenberg C, Aschoff P, Schanz S, et al. Prospective comparison of $18 \mathrm{~F}$-fluorodeoxyglucose positron emission tomography/ computed tomography and whole-body magnetic resonance imaging in staging of advanced malignant melanoma. Eur $\mathrm{J}$ Cancer 2007;43:557-64. 